\title{
Semiconductor Electrochemistry and Localised Corrosion
}

\author{
C.M. Rangel, ${ }^{*}$ M. da Cunha Belo ${ }^{a, b}$ \\ Instituto Nacional de Engenharia e Tecnologia Industrial, Electrochemistry of Materials, \\ DMTP-Paço do Lumiar, 22, 1649-038 Lisboa, Portugal \\ ${ }^{a}$ Instituto Superior Técnico, Departamento de Engenharia Química, Av. Rovisco Pais, \\ 1049-001 Lisboa, Portugal \\ ${ }^{b}$ Centre National de la Recherche Scientifique - CECM - 15 Rue Georges Urbain \\ F94407 Vitry-sur-Seine Cedex - France
}

Received 6 January 2004; accepted in revised form 1 August 2004

\begin{abstract}
In the present work, the electronic structure of the passive film formed on austenitic stainless steel of the 304 type and its implications on the initiation of localised corrosion are investigated taking into account concepts developed in semiconductor physics and semiconductor electrochemistry. Capacitance measurements (Mott-Schottky approach), show that the susceptibility of AISI 304 stainless steel to stress corrosion cracking (SCC) in boiling chloride containing aqueous solutions is closely linked to the formation of a chromium rich passive oxide film with p-type semiconductivity. A small polarisation is required to drastically change the electric field at the film-electrolyte interface, as a consequence of the high doping level of the passive film. Initiation of the SCC phenomenon is described as the consequence of localised changes in the semiconductive properties of the passive film.
\end{abstract}

Keywords: stress corrosion cracking, stainless steel, passive films, semiconductive properties.

\section{Introduction}

Stress corrosion cracking (SCC) promotes catastrophic failures on different engineering materials as a result of the combined synergistic interaction of mechanical, metallurgical and environmental factors. The number of works concerning research into these aspects is considerable, with emphases in fracture

\footnotetext{
* Corresponding author. E-mail address: carmen.rangel@ineti.pt
} 
morphology and electrochemical aspects within which the passive film and its rupture have been considered of utmost importance [1-5]. However, in spite of the vast amount of research efforts, the understanding of stress corrosion phenomena demands further investigation. For stainless steel the role of film composition, structure and properties associated to SCC have not been established.

It is the aim of the present work to demonstrate how the electronic structure of the film can provide information for the interpretation of localised corrosion processes such as SCC, taking into consideration that the passive state is related to the development of an oxide film with semiconductive character [6-9].

The study of the electronic structure of the passive film and the effect of polarisation on the film stability for AISI stainless steel 304 is carried out using the Mott-Schottky approach.

\section{Experimental}

Table 1 shows the composition of AISI 304 used in this work. The specimens were subjected to vacuum annealing (20 minutes at $1050{ }^{\circ} \mathrm{C}$ ) followed by rapid cooling also in vacuum.

Table 1. Elemental chemical composition of stainless steel AISI 304.

\begin{tabular}{lccccccc}
\hline Elements & $\mathrm{Cr}$ & $\mathrm{Ni}$ & $\mathrm{C}$ & $\mathrm{N}$ & $\mathrm{Mn}$ & $\mathrm{Si}$ & $\mathrm{Fe}$ \\
\hline wt $\%$ & 18 & 10 & 0.04 & 0.01 & 1 & 0.5 & balance
\end{tabular}

Specimens were mechanically polished with $\mathrm{SiC}$ paper up to 1200 grit, washed, cleaned ultrasonically, dried in air and kept in a desiccator until use. Before every experiment, specimens were electrochemically polished in an acetic-perchloric acid bath. The exposed area of the specimens was of $2 \mathrm{~cm}^{2}$.

Experiments were conducted in concentrated $\mathrm{MgCl}_{2}$ solution (slightly diluted in respect to the traditional $42 \mathrm{wt} \%$ solution) with a boiling point of $142{ }^{\circ} \mathrm{C}$. Stress corrosion cracking tests were performed at a constant load of $270 \mathrm{MPa}$. 
A study in a redox system was also conducted using a deaerated solution of $\mathrm{Na}_{2} \mathrm{SO}_{4}(0.5 \mathrm{M})+\mathrm{K}_{3} \mathrm{Fe}(\mathrm{CN})_{6}(0.1 \mathrm{M})+\mathrm{K}_{4} \mathrm{Fe}(\mathrm{CN})_{6}(0.1 \mathrm{M})$ at room temperature. A three-electrode configuration cell was used. All electrochemical potentials referred in this work are indicated relative to the saturated calomel electrode (SCE).

Data on the electronic properties of the passive film were obtained by capacitance measurements which reflect the charge distribution in the film. The relationship between the differential capacitance, $\mathrm{C}$, and the potential drop in the semiconductor can be written in the following form [10]:

$$
1 / C^{2}=\frac{2}{\varepsilon \varepsilon^{0} e N}\left(E-E_{f b}-k T / e\right)
$$

representing the Mott-Schottky equation, where e is the elementary charge $(+\mathrm{e}$ for electrons and - e for holes), $\varepsilon$ is the dielectric constant, $\varepsilon^{\mathrm{o}}$ the permittivity in vacuum, $\mathrm{N}$ is the acceptor or donor concentration, $\mathrm{E}$ is the applied potential, and $\mathrm{E}_{\mathrm{fb}}$, the flat band potential. Using eq. (1) it is possible, from the intersect with the potential axis of the $\mathrm{C}^{-2}$ versus $\mathrm{U}$ plot, to determine $\mathrm{E}_{\mathrm{fb}}$. The donor or acceptor concentrations can be estimated from the slopes of the straight lines obtained.

The Mott-Schottky plots were obtained by performing a potential scan in the cathodic direction.

The capacitance measurements were carried out at $3160 \mathrm{~Hz}$ within the potential range from +0.5 to $-0.5 \mathrm{~V}(\mathrm{SCE})$. A perturbing signal of $10 \mathrm{mV}$ rms was used. Potential was scanned by applying successive steps of $100 \mathrm{mV}$. EG\&G equipment was used throughout associated to a Lock-in Amplifier Model 5210. The capacitance was calculated using an RC series equivalent circuit network. AES analysis used a cylindrical mirror analyser and an axial gun. The primary beam had an incidence of $30^{\circ}$ relatively to the plan normal to the surface of the specimen and energy of $3 \mathrm{keV}$ and an incident angle of $50^{\circ}$ leading to an etching rate of approximately $0.5 \mathrm{nmmin}^{-1}$. 


\section{Results}

Stress corrosion cracking of stainless steels in boiling magnesium chloride solution is preceded by an incubation period which allows for an increase in the open circuit electrochemical potential of the system from $-\sim 0.45$ to $-\sim 0.30$ V(SCE).

Fig. 1a) shows the potentiodynamic current density (i) - potential (U) curves obtained for AISI 304 stainless steel in a boiling $\mathrm{MgCl}_{2}$ solution, at a scan rate of $20 \mathrm{mVs}^{-1}$. Also represented in the same figure, are the curves corresponding to the main alloying elements in AISI 304 stainless steel, such as Cr, Fe and Ni. It is evident that only the stainless steel shows a passive region, even though the potential domain is very restricted. The fact that the value of the current density corresponding to potentials in the vicinity of the open circuit appeared negative, may be indicative of the use of a relative fast scan rate. The potential domain for stress corrosion cracking is indicated in Fig. 1b), where data, obtained in the same experimental conditions, show the time to failure (fracture at constant load) for AISI 304 stainless steel as a function of potential.

The initiation of localised corrosion process, in this case stress corrosion cracking, is associated to potential conditions in which the formation of an oxide film promotes sufficiently low currents for a passive condition to be established together with the presence of a load that allows compatible stresses for the development of active cracks.

A study of the film composition, in situations that covered the transition from non-susceptible to susceptible potentials, was carried out using ex-situ AES analysis. Fig. 2 shows the depth composition profiles obtained for passive films formed on 304 stainless steels in $\mathrm{MgCl}_{2}$ solutions at - $0.45 \mathrm{~V}$ (Fig. 2a) and - 0.30 V (Fig. 2b). The analytical study demonstrated that the chemical composition of the film changes with potential rise. The film is composed by a nickel rich-iron mixed oxide in the first case, and by essentially a chromium-iron oxide when the specimens have been exposed to the corrosive medium at a susceptible potential (see Fig. 2b). In the present comparative study, it is assumed that, even if the first 
atomic superficial layers of the film are susceptible to contamination when in contact with air, the essential characteristics of the films are retained, so that global composition is not altered in a significant manner.

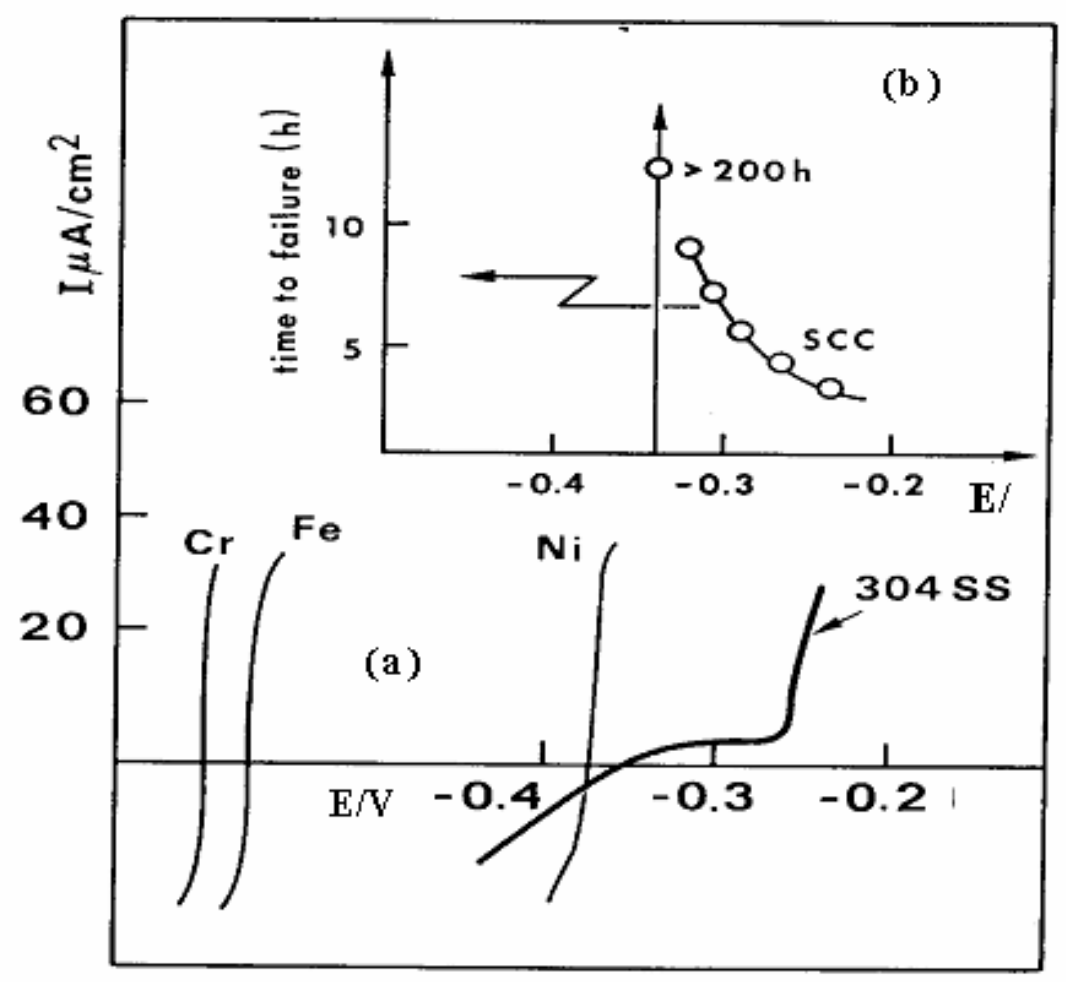

Figure 1. (a) Potentiodynamic current density - potential curves obtained for AISI 304 stainless steel in a concentrated boiling $\mathrm{MgCl}_{2}$ solution. Also represented are the curves corresponding to the main alloying elements in AISI 304 stainless steel in the same solution. (b) Potential domain for stress corrosion cracking is indicated by the time to failure versus potential curve obtained in the same experimental conditions.

A capacitance study was undertaken for specimens previously passivated in the $\mathrm{MgCl}_{2}$ boiling solution, in order to form the oxide film at the desired potential, with the measurements proper taken in a borate solution of $\mathrm{pH} 9.2$ (see experimental section). Results are presented in the form of Mott-Schottky plots.

The film formed at - $0.45 \mathrm{~V}(\mathrm{SCE})$ reveals n-type behaviour i.e, a positive slope in the $\mathrm{C}^{-2}$ versus $\mathrm{U}$ representation, see Fig. 3a, curve 1. Conversely, the film formed at a more positive potential (- $0.30 \mathrm{~V}(\mathrm{SCE}))$ reveals p-type behaviour. This is illustrated by the negative slope of the Mott- Schottky representation, see 
Fig. 3a, curve 2. It is suggested that $\mathrm{SCC}$ initiation in $\mathrm{MgCl}_{2}$ solutions is associated to the formation of a passive film with p-type semiconducting properties.
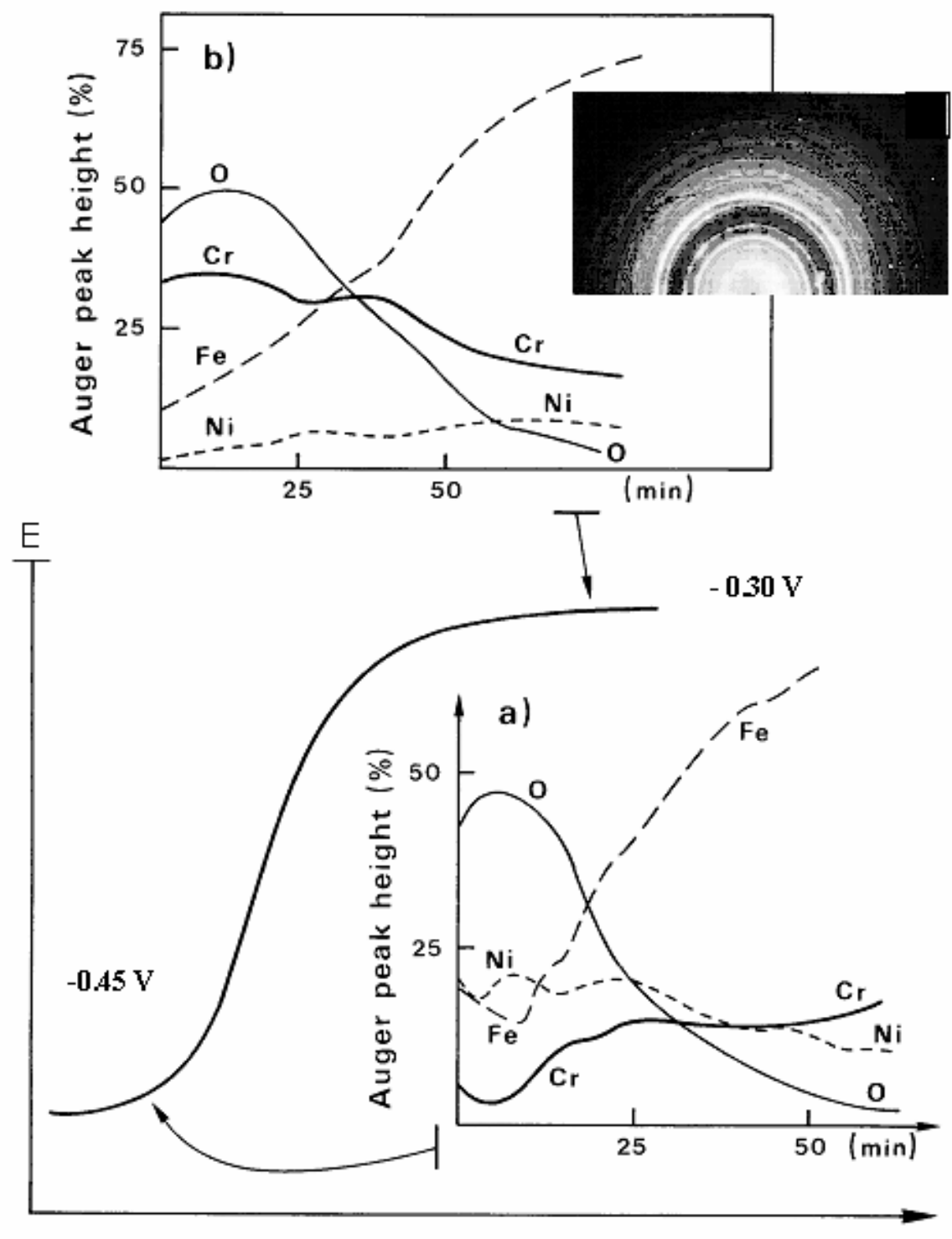

Time

Figure 2. Auger depth profiles for passive films on AISI 304 stainless steel in boiling $\mathrm{MgCl}_{2}$ solution formed at (a) $-0.45 \mathrm{~V}(\mathrm{SCE})$ and (b) $-0.30 \mathrm{~V}(\mathrm{SCE})$; electron diffraction pattern for the passive film (chromium rich spinel) is also shown.

The semiconducting character of the films formed on AISI 304 stainless steel in $\mathrm{MgCl}_{2}$ solution is confirmed by the electrochemical study conducted in a redox 
system (using a deaerated solution of $\mathrm{Na}_{2} \mathrm{SO}_{4}(0.5 \mathrm{M})+\mathrm{K}_{3} \mathrm{Fe}(\mathrm{CN})_{6}(0.1 \mathrm{M})+$ $\mathrm{K}_{4} \mathrm{Fe}(\mathrm{CN})_{6}(0.1 \mathrm{M})$ at room temperature, see experimental section).

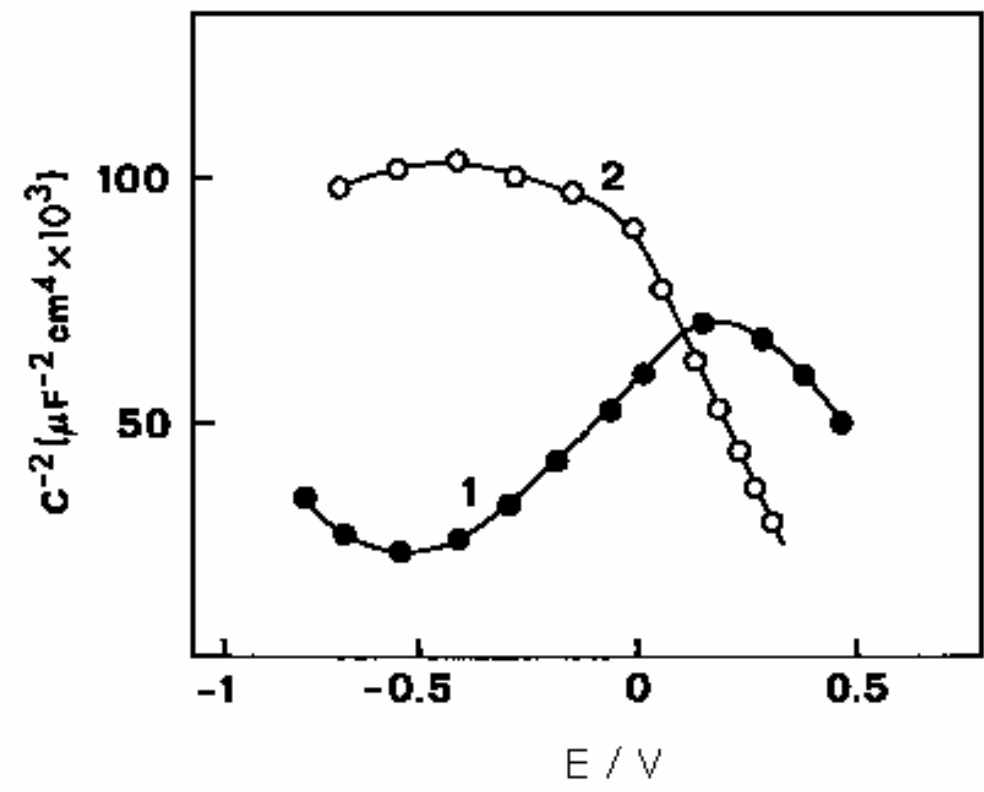

(a)

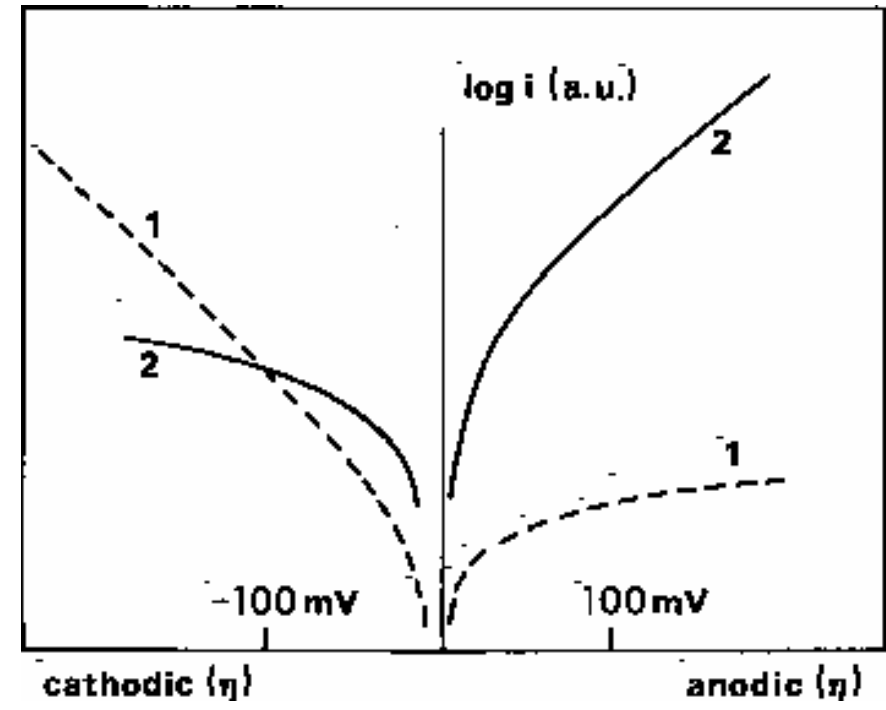

(b)

Figure 3. (a) $\mathrm{C}^{-2}$ versus applied potential, $\mathrm{U}$, for passive films formed on AISI stainless steel in boiling $\mathrm{MgCl}_{2}$ solution at $-0.45 \mathrm{~V}(\mathrm{SCE})$ (1) and - $0.30 \mathrm{~V}(\mathrm{SCE})$ (2). (b) The current density as a function of the overpotential $(\eta)$ obtained in the redox system (see experimental) for AISI 304 stainless steel previously passivated in a boiling $\mathrm{MgCl}_{2}$ solution at $-0.45 \mathrm{~V}(\mathrm{SCE})(1)$ and $-0.30 \mathrm{~V}(\mathrm{SCE})(2)$. 
It is well known that when a semiconductor electrode is exposed to a solution, the transfer of electronic charge depends on the mutual disposition of the characteristic energy levels for the conduction and valence bands. So, it can be assumed that the current voltage characteristics are markedly asymmetric [11]. In the case of an n-type semiconductor electrode the current increases exponentially under cathodic polarisation whilst remaining limited under anodic polarisation. Conversely, for a p-type semiconductor electrode it is the anodic current that increases exponentially under polarisation.

Fig. 3b) shows the current-potential curves obtained for the passive films formed during the incubation time (non-susceptible potential, - $0.45 \mathrm{~V}$ (curve 1)) and that formed at a susceptible potential, $-0.30 \mathrm{~V}$ (curve 2). As it can be observed, the electrochemical response can be related to the behaviour of an n-type and a ptype semiconductor, for curves 1 and 2 , respectively.

\section{Discussion}

The protective character of passivity can be explained by the development of passive oxide layers with semiconducting properties.

One of the characteristics of the system AISI 304 stainless steel / boiling $\mathrm{MgCl}_{2}$ solutions is the existence of a very small potential range for the passive state.

The present study demonstrated that there is a change in film composition associated to the transition to susceptible potentials for SCC. On the other hand, capacitance measurements showed that the plot $\mathrm{C}^{-2}$ versus $\mathrm{U}$ revealed the semiconducting behaviour of chromium oxide. Initially an iron-nickel rich spinel forms at lower potentials in this environment, while a chromium rich spinel formed at susceptible potentials.

It is well known that the electric field strength at a semiconductor electrolyte interface is normally much smaller than in the case of a metal-electrolyte interface. Therefore, the influence of electrostatic forces on the energy barriers for charge-transfer processes may be negligible. In place of this, the concentration of the electronic charge carriers in the surface of the semiconductor electrode varies by orders of magnitude with changing voltage and becomes an 
important factor for the rate of all reactions in which these charges carriers take place directly. It should be remarked that the characteristic feature of such current-voltage curves is that in the conduction band only the cathodic processes are influenced by applied voltage, whereas in the valence band only the anodic processes depend on the voltage [11].

When the electrochemical potential increases, there is a transition from n-type to p-type semiconductivity of the film (the possible defects are interstitials (iron and nickel ions) and oxygen vacancies in the first case, and chromium vacancies in the latter). This evolution of the electronic structure can be related to changes of both chemical composition and concentration of the predominant structural defects.

The passive films formed on 304 stainless steel in the specific environment under study, which promotes stress corrosion cracking, are highly doped and the Fermi energy is situated very close to the valence band energies. The presence of dislocations, as a result of the deformation process that leads to stress corrosion cracking, affects the energy of formation of charged defects during film growth and influences the donor density of the oxide film. For a p-type semiconductor, the number of vacancies (the doping defects in the case of chromium oxide are Cr vacancies) increases and the Fermi level may reach the valence band which corresponds to a situation of degeneracy. This means that the conduction approaches that of a simple metal electrode. From this phenomenon may result a local modification of the electric field and "chanelling" of the electronic carriers. The influence of dislocations is obviously accentuated in the regions of the metallic surface where slip planes intercept the passive film and a large number of dislocations may be concentrated.

\section{Conclusions}

- The susceptibility of AISI 304 stainless steel to stress corrosion cracking in boiling chloride containing aqueous solutions, is closely linked to the formation of a chromium rich passive oxide film with p-type semiconductivity. 
- The initiation of the SCC phenomenon can be described as being the consequence of a localised change of the semiconductivity properties of the passive film.

\section{References}

1. R.W. Staehle, NACE 5- "Stress Corrosion Cracking and Hydrogen Embrittlement of Iron-Based Alloys", Houston, TX: NACE, 1977.

2. P. Engsseth, J.C. Scully, Corros. Sci. 15 (1975) 505.

3. T. Kodoma, J.R. Ambrose, Corrosion 33 (1977) 155.

4. X.G. Zhang, J. Verrecken, Corrosion 45 (1989) 57.

5. H.S. Kwon, E.A. Cho, K.A. Yeom, Corrosion 56 (2000) 32.

6. A.D. Paola, F. Di Quarto, C. Sunseri, Corros. Sci. 26 (1986) 935.

7. K. Azumi, T. Ohtsuka, N. Sato, J. Electrochem. Soc. 133 (1986) 1326.

8. U. Stimming, Electrochim. Acta 31 (1986) 415.

9. C.M. Rangel, S. Faty, M. da Cunha Belo, Port. Electrochim. Acta 20 (2002) 119.

10. S.R. Morrison, Electrochemistry at Semiconductor and Oxidized Metal Electrodes, Chap. 5, Plenum Press, New York, 1980.

11. H. Gerisher, in: P. Delahay (Ed.), Advances in Electrochemistry and Electrochemical Engineering, Vol. I, Willey- Interscience, New York ,1961. 